Article

\title{
Application of Deep Eutectic Solvents to Prepare Mixture Extracts of Three Long-Lived Trees with Maximized Skin-Related Bioactivities
}

\author{
Yan Jin ${ }^{1}$, Dasom Jung ${ }^{1}, \mathrm{Ke} \mathrm{Li}^{1}{ }^{1}$, Keunbae Park ${ }^{1}$, Jaeyoung Ko ${ }^{2, *}$, Misuk Yang ${ }^{2}$ and \\ Jeongmi Lee ${ }^{1, *(D)}$ \\ 1 School of Pharmacy, Sungkyunkwan University, Suwon, Gyeonggi-do 16419, Korea \\ 2 Amorepacific Research and Development Center, Giheung-gu, Yongin 17074, Korea \\ * Correspondence: jaeyoungko@amorepacific.com (J.K.); jlee0610@skku.edu (J.L.); \\ Tel.: +82-31-290-7784 (J.L.); Fax: +82-31-292-8800 (J.L.)
}

Received: 20 May 2019; Accepted: 22 June 2019; Published: 26 June 2019

\begin{abstract}
This study aims to apply deep eutectic solvents (DESs) as safe and efficient extraction media that could yield maximized skin-related bioactivities from a mixture of long-lived trees. Ginkgo biloba L., Cinnamomum camphora (L.) J. Presl., and Cryptomeria japonica (L.f.) D. Don, native to Asia, were examined as potential resources of cosmeceutical products. Various DESs synthesized from cosmetics-compatible compounds were used to prepare leaf extracts. A DES containing glycerol and xylitol yielded the highest extractability for isoquercetin, and was selected as the optimal solvent. Isoquercetin has various bioactivities and was found in the extracts of the leaves of all three trees. Then, a series of mixtures of the tree leaves were prepared according to a simplex-centroid mixture design, and their DES-extracts were tested for skin-related activities, including antioxidant, anti-tyrosinase, and anti-elastase activities. The mixture design resulted in two special cubic models and one quadratic model best fitted for describing the antioxidant and anti-elastase activities, and the anti-tyrosinase activity, respectively. Based on the established models, three different optimal formulations of the three kinds of tree leaves were suggested for maximized responses. The present strategy, which is based on the simplex-centroid mixture design with a DES as the extraction solvent, could be applied to developing new materials from a mixture of natural resources, suitable for the cosmetics and related fields.
\end{abstract}

Keywords: cosmeceutical product; long-lived trees; simplex-centroid mixture design; deep eutectic solvents; bioactivity

\section{Introduction}

The skin can be damaged by numerous factors including ultraviolet radiation, smoking, hormonal imbalance, ethanol ingestion, air pollution, and inflammation [1,2]. Cosmetics are most relied upon for skin damage prevention because they are more convenient and economical, and have fewer side effects than pharmaceuticals or cosmetic surgery [3]. Thus, this has increased the demand for effective cosmeceutical products containing natural bioactive compounds [4].

The need for natural bioactive compound-containing cosmeceutical products might be met by a certain category of plants containing compounds with useful bioactivities in the human skin. Asian countries, like Korea, Japan, and China, have always used traditional medicines for various diseases, and have been at the forefront of cosmeceutical product production from natural resources [5]. By actively searching for long-lived trees native to Asia that may contain compounds with skin-beneficial effects, we found Ginkgo biloba L. (GB), Cinnamomum camphora (L.) J. Presl. (CC), 
and Cryptomeria japonica (L.f.) D. Don (CJ). The living fossil GB has continuously gained popularity as a dietary supplement [6] and complementary medicine [7]. Extracts of its leaves are rich in ginkgolides, flavonoids, biflavones, terpenoids, and polyphenols [8,9], and have been used for treating age-related diseases [10]. GB is also used in anti-aging cosmetics due to its antioxidant, anti-lipid peroxidation, and anti-inflammatory activities [11]. The evergreen tree CC, commonly known as the camphor tree, is used as a medicinal plant [12], as it exhibits a number of biological activities, such as antioxidant, anti-inflammatory [13], and antifungal [14], owing to its bioactive compounds such as terpenoids and phenylpropanoids. CJ, commonly called the Japanese cedar, is known to have strong antimicrobial [15], anti-inflammatory [16], and antifungal [17] activities. It also has skin-whitening and antioxidant activities based on anti-tyrosinase, radical scavenging, and superoxide dismutase assay results [18]. We hypothesized that a mixture of the leaf extracts of these three trees could synergistically exert skin-beneficial effects. The skin-related bioactivities of tree extracts are likely to be associated with polyphenols. Because their extraction yields and compositions can vary greatly depending on a number of factors, including solvent type and concentration, temperature, time, and extraction method, the extraction conditions need to be carefully optimized, as reported by Tanase et al. [19].

The versatile green solvents, deep eutectic solvents (DESs), have recently gained much attention due to their low toxicity, high extractability, and good biodegradability. Their potential usage in various fields, such as the cosmetics industry, has been suggested [20,21]. Therefore, the current study aims at applying DESs as safe and efficient extraction mediums that could yield maximized skin-related bioactivities from a mixture of the three long-lived trees. We therefore synthesized a number of DESs using components that are permissible according to the European Commission's Inventory of Cosmetic Ingredients (2006) [22], and compared their extraction efficiency for a selected marker compound. Then, we applied a simplex-centroid mixture design to locate the optimal combinations of CC, CJ, and GB that would maximally allow beneficial skin-related bioactivities, including antioxidant, anti-tyrosinase, and anti-elastase activities. This study suggests the potential of a mixture of the three extracts in DESs as a new natural bioactive compound-containing material for cosmeceutical product development.

\section{Materials and Methods}

\subsection{Chemicals and Reagents}

Fresh leaves of Cinnamomum camphora (L.) J. Presl., Cryptomeria japonica (L.f.) D. Don, and Ginkgo biloba L. were collected in Hanlim-eup, Aewol-eup, and Hawon-dong, respectively, all of which are local villages in Jeju Island, Republic of Korea. The samples were collected in October, 2018 and identified botanically by Dr. Jun Hwan Shin (Dongyang University, Yeongju, Korea). Three voucher specimens (CC: AP-0100, CJ: AP-0101, and CB: AP-0103) were deposited at the Plant Archive of Amorepacific Research and Development Center. Dried leaves of CC, CJ, and GB trees were ground into fine powders using an electric mill and stored in sealed containers at $4{ }^{\circ} \mathrm{C}$ until use. The pulverized samples were directly used for extraction without sieving. The compounds used for DES synthesis included glycerol ( $\geq 99.5 \%)$, xylitol ( $\geq 99 \%)$, D-(+)-glucose ( $\geq 99.5 \%)$, D-sorbitol ( $\geq 99.5 \%)$, D- $(+)$-maltose $(\geq 99 \%)$, maltitol $(\geq 98 \%)$, D-(-)-fructose $(\geq 99 \%)$, sucrose $(\geq 99.5 \%)$, betaine ( $\geq 98 \%)$, DL-malic acid $(\geq 99 \%)$, and DL-lactic acid ( $\sim 90 \%$ ) (Sigma-Aldrich, St. Louis, MO, USA). They were used without further purification. The following were used for the activity assays: 2,2-di(4-tert-octylphenyl)-1-picrylhydrazyl (DPPH) and 6-hydroxy-2,5,7,8-tetramethyl chroman-2-carboxylic acid (Trolox; 97\%) for the antioxidant activity; tyrosinase from mushroom, levodopa (L-DOPA), sodium phosphate dibasic, sodium phosphate monobasic, and kojic acid (KA, $\geq 99 \%$ ) for the anti-tyrosinase activity; and elastase from porcine pancreas, N-succinyl-Ala-Ala-Ala-p-nitroanilide (SANA), tris base, and hydrochloric acid for the anti-elastase activity (all; Sigma-Aldrich). Isoquercetin (ISO, > 98\%) (Biopurify Phytochemicals Ltd., Chengdu, China) and HPLC-grade methanol, water, and acetonitrile were also purchased (Honeywell Burdick \& Jackson, Ulsan, Korea). 


\subsection{Sample Preparation}

Tree powders $(60 \mathrm{mg})$ were each extracted in $1 \mathrm{~mL}$ of extraction solvent with ultrasonic irradiation for $60 \mathrm{~min}$. For this, an ultrasonic bath (Powersonic 410) from Hwashin Technology (Seoul, Korea) was used without temperature control. After the extraction, the bath temperature increased by approximately $20^{\circ} \mathrm{C}$. After centrifugation at $12300 \times g$ for $10 \mathrm{~min}$, the supernatants were collected for further analyses. For ISO content determination, each supernatant was diluted (5-fold) with $70 \%$ methanol, and filtered through a $0.45 \mu \mathrm{m}$ pore membrane syringe filter before injection into LC-UV. For the antioxidant and anti-elastase activity assays, each supernatant was diluted (10-fold) in $70 \%$ methanol, while for the anti-tyrosinase activity assay, a 10-fold diluted extract was prepared with 0.1 M phosphate buffer ( $\mathrm{pH}$ 6.8).

\subsection{Synthesis of DESs}

The DESs were synthesized using the previously reported heating method $[23,24]$, with slight modifications. In brief, accurately weighed individual components were mixed with/without a minimum amount of water and stirred at $80{ }^{\circ} \mathrm{C}$. Then, the mixtures were kept at $-80^{\circ} \mathrm{C}$ for $2 \mathrm{~h}$, followed by freeze drying until reaching a constant weight. All 15 DESs in Table 1 could be synthesized as homogeneous transparent liquids.

Table 1. List of synthesized deep eutectic solvents (DESs).

\begin{tabular}{|c|c|c|c|c|}
\hline DES No. & Component 1 & Component 2 & Component 3 & Molar Ratio \\
\hline $1^{\mathrm{a}}$ & \multirow{9}{*}{ Glycerol } & Xylitol & & $2: 1$ \\
\hline $2^{a}$ & & Maltose & & $3: 1$ \\
\hline $3^{a}$ & & Sorbitol & & $2: 1$ \\
\hline $4^{\mathrm{a}}$ & & Fructose & & $3: 1$ \\
\hline $5^{b}$ & & Sucrose & & $3: 1$ \\
\hline $6^{b}$ & & Glucose & & $3: 1$ \\
\hline $7^{b}$ & & Maltitol & & $3: 1$ \\
\hline $8^{b}$ & & Malic acid & & $1: 1$ \\
\hline $9^{b}$ & & Malic acid & & $1: 2$ \\
\hline $10^{b}$ & Lactic acid & Glucose & & $1: 2$ \\
\hline $11^{\mathrm{b}}$ & \multirow{2}{*}{ Fructose } & Sucrose & & $1: 1$ \\
\hline $12^{b}$ & & Sucrose & Glucose & 1:1:1 \\
\hline $13^{b}$ & \multirow{3}{*}{ Betaine } & Sucrose & & $1: 1$ \\
\hline $14^{\mathrm{b}}$ & & Sucrose & & $1: 2$ \\
\hline $15^{b}$ & & Glucose & & $1: 1$ \\
\hline
\end{tabular}

a Synthesized without water. ${ }^{b}$ Synthesized using a minimum amount of water before lyophilization.

\subsection{Determination of ISO as a Common Marker Compound for Extraction Efficiency Using LC-UV}

Chemical profiles of the leaf extracts of the three trees were obtained using liquid chromatography-diode array detection (LC-DAD) (Figure S1), which indicated that they had different constituents and compositions. The UV spectra of the peaks in the chromatograms showed they contained a number of flavonoids, a class of components widely existing in plants, with various beneficial bioactivities (e.g., characteristic $\lambda_{\max }$ values at 254-264 nm and 347-353 nm). In particular, the flavonoid isoquercetin (ISO) could be identified in the extracts of all three tree leaves (Figure S1). Owing to its many beneficial bioactivities, including antioxidant [25], antivirus [26], and anti-inflammation [27] activities, ISO could serve as a marker for assessing the extraction efficiency of various extraction solvents. 
A stock solution of ISO $(1000 \mu \mathrm{g} / \mathrm{mL})$ was prepared in methanol, and used to prepare a series of standard working solutions with concentrations ranging from $1-100 \mu \mathrm{g} / \mathrm{mL}$ in $70 \%$ methanol. The solutions were stored at $4{ }^{\circ} \mathrm{C}$ until use.

A Waters HPLC system (Waters Co., Milford, MA, USA) equipped with a Waters 2695 separation module and a Waters 996 photodiode array detector was used to determinate ISO levels. The injection volume was $10 \mu \mathrm{L}$, and chromatographic separation was achieved on a Gemini $\mathrm{C}_{18}$ column $(250 \times 4.6 \mathrm{~mm}, 5 \mu \mathrm{m}$; Phenomenex, Torrance, CA, USA) at room temperature. The detector wavelength was set at $354 \mathrm{~nm}$. The mobile phase consisted of eluent A ( $1 \%$ formic acid in water) and eluent B ( $1 \%$ formic acid in acetonitrile) run at a flow rate of $1 \mathrm{~mL} / \mathrm{min}$. Gradient elution was performed as follows: $0-3 \mathrm{~min}, 5-20 \%$ of $\mathrm{B} ; 3-30 \mathrm{~min}, 15-20 \%$ of $\mathrm{B} ; 30-31 \mathrm{~min}, 20-100 \%$ of $\mathrm{B} ; 31-37 \mathrm{~min}, 100 \%$ of $\mathrm{B}$; and $\mathrm{B} \%$ was returned to $5 \%$ in $1 \mathrm{~min}$. The established calibration equation was $\mathrm{y}=20076 \mathrm{x}+1013\left(\mathrm{r}^{2}=1.0000\right.$, $\mathrm{n}=3)$.

\subsection{Antioxidant Activity Assay}

DPPH free radical scavenging activity was assessed using the previously established protocol [23]. Briefly, the supernatant was diluted (10-fold) in $70 \%$ methanol, and mixed with an equal volume of freshly prepared $0.2 \mathrm{mM}$ DPPH in methanol. Absorbance at $517 \mathrm{~nm}$ was measured after a $30 \mathrm{~min}$ incubation of the mixture seeded in a 96-well microplate in the dark at room temperature. The antioxidant abilities of the extracts were calculated using the following equation:

$$
\text { Scavenging activity } \%=\left(\mathrm{A}_{\text {control }}-\mathrm{A}_{\text {sample }}\right) / \mathrm{A}_{\text {control }} \times 100
$$

The radical scavenging activity was expressed as Trolox equivalents (mg TE/g tree leave sample). The linear calibration range for Trolox was $1-20 \mu \mathrm{g} / \mathrm{mL}$, with $\mathrm{y}=4.160 \mathrm{x}+2.955\left(\mathrm{r}^{2}=0.9982, \mathrm{n}=3\right)$.

\subsection{Anti-Tyrosinase Activity Assay}

The anti-tyrosinase activities of diluted samples (10-fold) were measured with L-DOPA as the substrate, as previously reported with slight modifications [28]. A mixture of $100 \mu \mathrm{L}$ of the test samples and $100 \mu \mathrm{L}$ of $80.61 \mathrm{U} / \mathrm{mL}$ of tyrosinase in $0.1 \mathrm{M}$ phosphate buffer ( $\mathrm{pH}$ 6.8) was incubated at room temperature for $15 \mathrm{~min}$. Then, $50 \mu \mathrm{L}$ of $2.5 \mathrm{mM}$ L-DOPA was added and the mixture was incubated at room temperature for $20 \mathrm{~min}$. Absorbance was measured at $475 \mathrm{~nm}$. Enzyme inhibitory activity was calculated using the following equation:

$$
\text { Inhibition } \%=\left(1-\left(\mathrm{A}_{1}-\mathrm{A}_{2}\right) /\left(\mathrm{A}_{3}-\mathrm{A}_{4}\right)\right) \times 100
$$

where, $A_{1}, A_{2}, A_{3}$, and $A_{4}$ are the absorbances of test sample with enzyme, test sample without enzyme, enzyme without test sample, and solution without test sample and enzyme, respectively.

\subsection{Anti-Elastase Activity Assay}

SANA was used as a substrate in the anti-elastase activity assay, conducted as previously reported with minor modifications [29]. First, $50 \mu \mathrm{L}$ of 10-fold diluted test sample was mixed with $100 \mu \mathrm{L}$ of $10 \mu \mathrm{g} / \mathrm{mL}$ elastase in $0.2 \mathrm{M}$ Tris- $\mathrm{HCl}$ buffer ( $\mathrm{pH} 8.0$ ), and incubated at room temperature for $15 \mathrm{~min}$. Then, $100 \mu \mathrm{L}$ of substrate (1.6 mM SANA dissolved in $0.2 \mathrm{M}$ Tris- $\mathrm{HCl}$ buffer, $\mathrm{pH}$ 8.0) was added to the mixture and incubated for $20 \mathrm{~min}$. Absorbance was measured at $405 \mathrm{~nm}$, and Equation (2) was used to calculate \% inhibition. All activity assays were performed in triplicate.

\subsection{Experimental Design and Statistical Analyses}

Design Expert 8.0 software (Statease Inc., Minneapolis, MN, USA) was used for the simplex-centroid mixture design, and for analyzing data and creating graphs. All experiments 
were performed in random order. Other statistical analyses, including multiple comparisons, were conducted using GraphPad Prism 6 for Windows (GraphPad Software, San Diego, CA, USA).

\section{Results and Discussion}

\subsection{Preparation of DESs}

A list of components was selected from the European Commission's Inventory of Cosmetic Ingredients (2006), considering their stability, price, and safety, and then combined at proper molar ratios with hydrogen bond acceptors (HBAs) and hydrogen bond donors (HBDs), based on the available literature $[20,23,24]$. As a result, a total of 15 DESs were successfully synthesized using the heating method (Table 1).

\subsection{Selection of DESs for Mixture Design Studies Based on the ISO Extraction Yields}

The ISO content of CC, CJ, and GB were determined to be 861,276 , and $118 \mu \mathrm{g} / \mathrm{g}$, respectively, when using $70 \% \mathrm{w} / \mathrm{w}$ DES 1 . Because CC had the highest ISO level among the three trees, it was therefore used as a model for comparing the extraction efficiency of the prepared DESs. The ISO levels post extraction with DESs 1-11 $(746-915 \mu \mathrm{g} / \mathrm{g})$ were significantly $(p<0.05)$ higher than those with hot water $(705 \mu \mathrm{g} / \mathrm{g})$ (Figure 1). However, they were significantly $(p<0.05)$ lower than those acquired using $70 \%$ methanol $(1086 \mu \mathrm{g} / \mathrm{g})$. Glycerol generally served as a good component for DESs that are effective for ISO extraction, whether its counterpart was a sugar or an organic acid. Moreover, organic acids appeared to be superior components to glycerol, and DESs 8-10 containing malic acid or lactic acid resulted in the highest ISO extraction yields. However, DESs with organic acids are usually highly viscous and thus difficult to handle [24], and so were the malic acid-based DESs (DESs 8 and 9). DES 10 , with yield similar to those of DESs 8 and 9, was excluded from further consideration because of the low lactic acid purity ( 90\%). Accordingly, DES 1 (ISO yield, $861 \mu \mathrm{g} / \mathrm{g}$ ), which could be synthesized from glycerol and xylitol and handled with ease, was deemed the reasonable extraction solvent for the tree leaves.

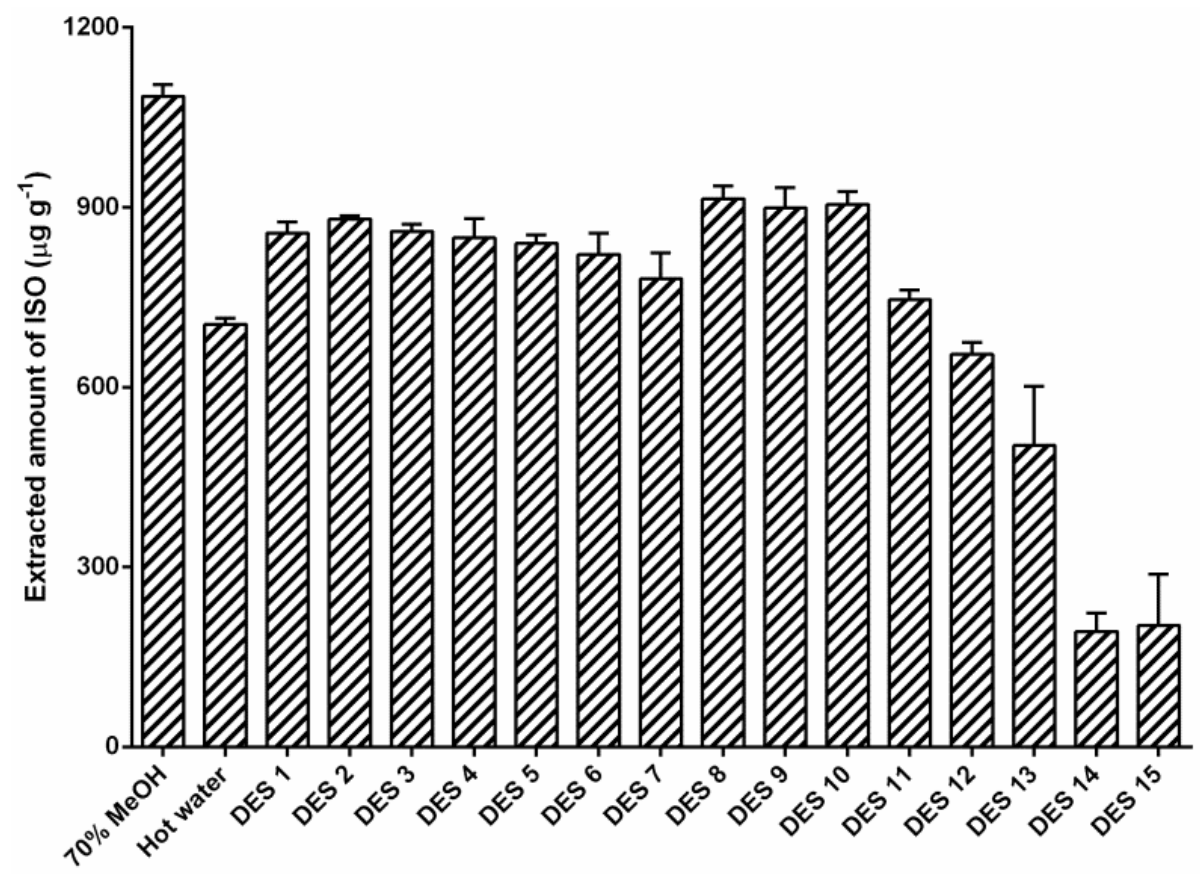

Figure 1. Comparison of isoquercetin (ISO) levels post extraction with DESs, to those post extraction with $70 \%$ methanol and water. The water content of all the tested DESs was $30 \% \mathrm{w} / \mathrm{w}$. Error bars indicate standard error of the mean $(n=3)$. 
Before applying DES 1 to the mixture design studies, the effects of several factors on extraction efficacy were examined. First, in varying the molar ratio of glycerol to xylitol from 1:1 to 5:1, at 30\% water, we found that DES 1 with a 5:1 ratio (designated as DES 1-5) had the highest efficiency, although it was not significantly different from that of DES 1 with a 4:1 ratio (DES 1-4) (Table S1). Then, the effect of the water content of DES 1 was assessed by measuring ISO yields with DES 1 with various water contents $(10-50 \% \mathrm{w} / \mathrm{w})$. We found that $30 \%$ water exhibited the highest yield $(946 \mu \mathrm{g} / \mathrm{g})$, while 50 and $10 \%$ water resulted in $<900 \mu \mathrm{g} / \mathrm{g}$ and $\sim 700 \mu \mathrm{g} / \mathrm{g}$, respectively (Table S2). Based on the faster synthesis time and lower viscosity, DES 1-5 was regarded as desirable, and its $70 \%$ aqueous solution was used as the final extraction solvent in the mixture design studies. It is apparent that the selected solvent $(70 \%$ $\mathrm{w} / \mathrm{w}$ DES 1-5) is less economical than water as an extraction medium. However, it is noteworthy that the extraction efficiency of the selected solvent was achieved without any heat treatment, and that the individual DES components can act as functional ingredients in cosmetics [20]. The water extraction, which had to be conducted at a boiling temperature for improved efficiency, might cause degradation of unstable compounds during extraction. These factors could provide merit for the use of the DES solution in this study.

\subsection{Simplex-Centroid Mixture Design}

Using the generated design matrix, nine different mixtures were prepared with $\mathrm{CC}, \mathrm{CJ}$, and GB, extracted in 70\% DES 1-5, and their antioxidant $\left(Y_{1}\right)$, anti-tyrosinase $\left(Y_{2}\right)$, and anti-elastase $\left(Y_{3}\right)$ activities were evaluated. The design matrix with coded and real values and the resulting responses is summarized in Table 2. For better evaluation of the design, the central point was repeated thrice $[30,31]$.

Table 2. Simplex-centroid mixture design and the resulting responses.

\begin{tabular}{ccccccc}
\hline \multirow{2}{*}{ No. } & \multicolumn{3}{c}{ Independent Variables $^{\mathbf{a}}$} & \multicolumn{3}{c}{ Dependent Variables } \\
\cline { 2 - 7 } & $\mathbf{X}_{\mathbf{1}} \mathbf{( m g )}$ & $\mathbf{X}_{\mathbf{2}} \mathbf{( m g )}$ & $\mathbf{X}_{\mathbf{3}} \mathbf{( m g )}$ & $\mathbf{Y}_{\mathbf{1}}$ & $\mathbf{Y}_{\mathbf{2}}$ & $\mathbf{Y}_{\mathbf{3}}$ \\
\hline 1 & $1(60)$ & $0(0)$ & $0(0)$ & 3.259 & 60.44 & 72.11 \\
2 & $0(0)$ & $1(60)$ & $0(0)$ & 3.175 & 68.35 & 78.86 \\
3 & $0(0)$ & $0(0)$ & $1(60)$ & 3.175 & 39.61 & 50.67 \\
4 & $0.5(30)$ & $0.5(30)$ & $0(0)$ & 3.246 & 50.55 & 76.96 \\
5 & $0.5(30)$ & $0(0)$ & $0.5(30)$ & 3.252 & 62.48 & 82.92 \\
6 & $0(0)$ & $0.5(30)$ & $0.5(30)$ & 3.131 & 30.50 & 9.83 \\
7 & $0.33(20)$ & $0.33(20)$ & $0.33(20)$ & 3.271 & 54.99 & 62.64 \\
8 & $0.33(20)$ & $0.33(20)$ & $0.33(20)$ & 3.252 & 50.98 & 72.07 \\
9 & $0.33(20)$ & $0.33(20)$ & $0.33(20)$ & 3.258 & 42.50 & 73.04 \\
\hline
\end{tabular}

${ }^{\mathrm{a}} \mathrm{X}_{1}$, Cinnamomum camphora (CC); $X_{2}$, Cryptomeria japonica (CJ); $X_{3}$, Ginkgo biloba (GB). ${ }^{\mathrm{b}} Y_{1}$, antioxidant activity (mg

$\mathrm{TE} / \mathrm{g}) ; Y_{2}$, anti-tyrosinase activity (\% inhibition); $Y_{3}$ : anti-elastase activity (\% inhibition).

The fitness of the linear, quadratic, and special cubic models for the three responses were analyzed (Table S3). The models with highest $\mathrm{R}^{2}$ adj values with statistical significance $(p<0.05)$ were selected for each response, resulting in the special cubic, quadratic, and special cubic models for $Y_{1}, Y_{2}^{3}$, and $Y_{3}$, respectively. The model equations are displayed in Table 3 , and the model qualities evaluated using analysis of variance (ANOVA) are summarized in Table S4.

Table 3. Model equations describing response as a function of mixture composition.

\begin{tabular}{cccc}
\hline Response $^{\mathrm{a}}$ & Model & $\mathbf{R}^{\mathbf{2}}$ & $\mathbf{R}^{\mathbf{2}}{ }_{\text {adj }}$ \\
\hline$Y_{1}$ & $3.26 \mathrm{X}_{1}+3.17 \mathrm{X}_{2}+3.17 \mathrm{X}_{3}+0.12 \mathrm{X}_{1} \mathrm{X}_{2}+0.14 \mathrm{X}_{1} \mathrm{X}_{3}-0.18 \mathrm{X}_{2} \mathrm{X}_{3}+1.31 \mathrm{X}_{1} \mathrm{X}_{2} \mathrm{X}_{3}$ & 0.9905 & 0.9619 \\
$Y_{2}^{3 \mathrm{~b}}$ & $219231.94 \mathrm{X}_{1}+317719.32 \mathrm{X}_{2}+60577.95 \mathrm{X}_{3}-531992.97 \mathrm{X}_{1} \mathrm{X}_{2}+441173.67 \mathrm{X}_{1} \mathrm{X}_{3}-$ & 0.9400 & 0.8399 \\
$Y_{3}$ & $617876.6 \mathrm{X}_{2} \mathrm{X}_{3}$ & 0.9911 & 0.9645 \\
\hline
\end{tabular}

a $Y_{1}$, antioxidant activity; $Y_{2}$, anti-tyrosinase activity; $Y_{3}$, anti-elastase activity. ${ }^{\mathrm{b}}$ Transformation: Power (Lambda $=$

3; constant, $\mathrm{k}=0$ ). 
For the antioxidant activity $\left(Y_{1}\right)$, only a special cubic model was significant ( $95 \%$ confidence level). The linear terms and cubic term had significant positive effects on the antioxidant activity (Table S4). As displayed in the contour plot (Figure 2a) and the model equation (Table 3), the $X_{1}$ variable (CC) provided a greater contribution than $X_{2}(\mathrm{CJ})$ and $X_{3}(\mathrm{~GB})$. Specifically, higher responses were observed as the $X_{1}$ portion of the mixture increased. The model yielded the following optimized proportions: $X_{1}, 53.3 \% ; X_{2}, 23.3 \%$; and $X_{3}, 23.3 \%$.
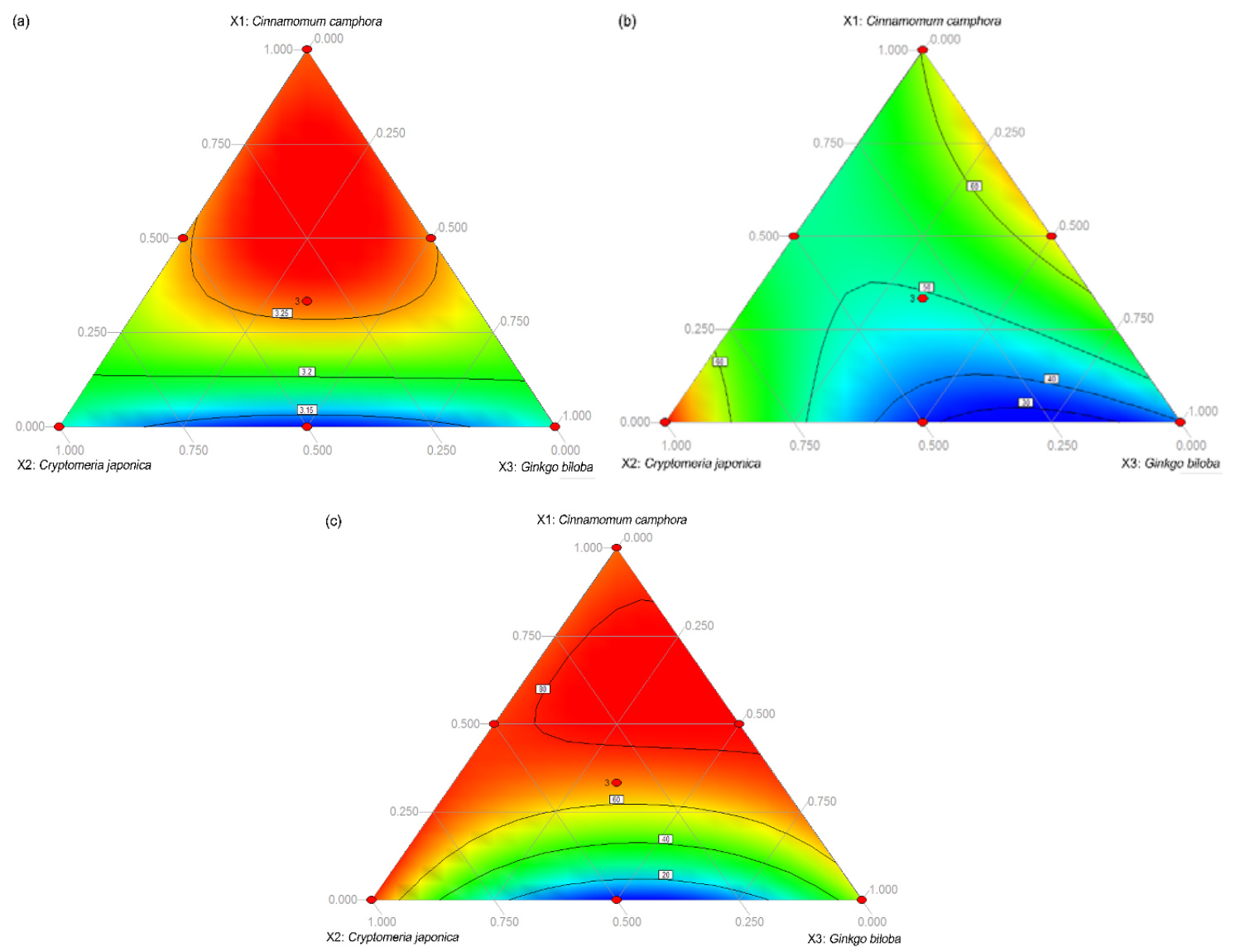

Figure 2. Response surface contour plots for the (a) antioxidant, (b) anti-tyrosinase, and (c) anti-elastase activities of different compositions of $X_{1}(\mathrm{CC}), X_{2}(\mathrm{CJ})$, and $X_{3}(\mathrm{~GB})$.

For the anti-tyrosinase activity $\left(Y_{2}\right)$, response transformation was vital to obtaining a significant model with no significant lack of fit, using the transformation type "power" with lambda and constant $k$ set as +3 and 0 , respectively. The resulting quadratic model was significant, in which only the two quadratic terms $X_{1} X_{2}$ and $X_{2} X_{3}$ were significant, as the term $X_{1} X_{2} X_{3}$ was excluded from modeling due to its negligible contribution (Table S4). $X_{2}$ (CJ) generally enhances the anti-tyrosinase activity, while $X_{1}$ and $X_{2}$, and $X_{2}$ and $X_{3}$ inhibit the anti-tyrosinase activity (Figure $2 \mathrm{~b}$ and Table 3 ). The model suggested the use of $100 \% X_{2}$ to achieve the highest anti-tyrosinase activity.

For the anti-elastase activity, only a special cubic model was significant, of which the linear terms, the quadratic term $X_{2} X_{3}$, and the cubic term were significant (Table S4). $X_{1}$ (CC) had the highest anti-elastase activity-enhancement effect, while the mixture of $X_{2}$ and $X_{3}$ inhibited anti-elastase activity (Figure $2 \mathrm{c}$ and Table 3). According to the model, the optimal compositions for achieving the highest anti-elastase activity were $63.6,14.1$, and $22.3 \%$ for $X_{1}, X_{2}$, and $X_{3}$, respectively.

\section{Conclusions}

The skin-beneficial bioactivities of the mixtures of extracts from three kinds of long-lived trees were investigated to identify potential new cosmeceutical product materials. Extracts of their leaves extracted 
using DESs contained cosmetics-compatible compounds, allowing safe and efficient ISO extraction into the extracts and their direct application in cosmetic formulations. Various mixtures of the three leaf extracts were prepared and systematically analyzed according to the simplex-centroid mixture design, providing information about the effects of the individual leaves on the three different bioactivities, as well as the optimal compositions of the three leaves for maximized antioxidant, anti-tyrosinase, and anti-elastase activities. The strategy used in this study could be applied to exploring new, effective, and safe materials for the cosmetics and related fields. However, further investigation of potential toxicity profiles for the selected DES would be needed before application, because DESs could have different toxicity than their individual components that have no or low toxicity.

Supplementary Materials: The following are available online at http://www.mdpi.com/2076-3417/9/13/2581/s1, Table S1. Effects of the molar ratios between glycerol and xylitol on the ISO yields; Table S2. Effects of the added water in DES 1-5 (glycerol:xylitol, 5:1); Table S3. Fit summary of the models; Table S4. ANOVA results of the established models for each response; Figure S1. Chromatographic profiles of the three extracts of (a) Ginkgo biloba, (b) Cinnamomum camphora, and (c) Cryptomeria japonica leaves prepared using 70\% w/w DES 1. Peak identification: $\mathrm{ISO}$, isoquercetin.

Author Contributions: Y.J., M.Y., J.K., and J.L. conceived and designed the experiments; Y.J., D.J., K.L., M.Y., J.K., and K.P. performed the experiments. Y.J. analyzed the data and drafted the manuscript; J.L. revised and edited the manuscript.

Funding: This work was financially supported by a grant from the Amorepacific Corporation (grant No.ORT-01-R18E999005) within the Amorepacific Open Research Program.

Conflicts of Interest: The authors declare no conflict of interest. J.K. and M.Y. work for Amorepacific Corporation.

\section{References}

1. Masaki, H. Role of antioxidants in the skin: Anti-aging effects. J. Dermatol. Sci. 2010, 58, 85-90. [CrossRef] [PubMed]

2. McDaniel, D.; Farris, P.; Valacchi, G. Atmospheric skin aging-Contributors and inhibitors. J. Cosmet. Dermatol. 2018, 17, 124-137. [CrossRef] [PubMed]

3. Shivanand, P.; Nilam, M.; Viral, D. Herbs play an important role in the field of cosmetics. Int. J. PharmTech Res. 2010, 2, 632-639.

4. Juhász, M.L.; Levin, M.K.; Marmur, E.S. The use of natural ingredients in innovative Korean cosmeceuticals. J. Cosmet. Dermatol. 2018, 17, 305-312. [CrossRef]

5. Eyles, A.; Bonello, P.; Ganley, R.; Mohammed, C. Induced resistance to pests and pathogens in trees. New Phytol. 2010, 185, 893-908. [CrossRef] [PubMed]

6. Ding, S.; Dudley, E.; Plummer, S.; Tang, J.; Newton, R.P.; Brenton, A.G. Quantitative determination of major active components in Ginkgo biloba dietary supplements by liquid chromatography/mass spectrometry. Rapid Commun. Mass Spectrom. 2006, 20, 2753-2760. [CrossRef] [PubMed]

7. Shu, Z.; Shar, A.H.; Shahen, M.; Wang, H.; Alagawany, M.; El-Hack, M.E.A.; Kalhoro, S.A.; Rashid, M.; Shar, P.A. Pharmacological Uses of Ginkgo Biloba Extracts for Cardiovascular Disease and Coronary Heart Diseases. Int. J. Pharmacol. 2019, 15, 1-9.

8. van Beek, T.A.; Montoro, P. Chemical analysis and quality control of Ginkgo biloba leaves, extracts, and phytopharmaceuticals. J. Chromatogr. A 2009, 1216, 2002-2032. [CrossRef] [PubMed]

9. van Beek, T.A. Chemical analysis of Ginkgo biloba leaves and extracts. J. Chromatogr. A 2002, 967, 21-55. [CrossRef]

10. Zuo, W.; Yan, F.; Zhang, B.; Li, J.; Mei, D. Advances in the studies of Ginkgo biloba leaves extract on aging-related diseases. Aging Dis. 2017, 8, 812. [CrossRef]

11. Chan, P.-C.; Xia, Q.; Fu, P.P. Ginkgo biloba leave extract: Biological, medicinal, and toxicological effects. J. Environ. Sci. Health C 2007, 25, 211-244. [CrossRef] [PubMed]

12. Chen, W.; Vermaak, I.; Viljoen, A. Camphor-A fumigant during the black death and a coveted fragrant wood in ancient Egypt and Babylon-A review. Molecules 2013, 18, 5434-5454. [CrossRef] [PubMed]

13. Li, Y.-R.; Fu, C.-S.; Yang, W.-J.; Wang, X.-L.; Feng, D.; Wang, X.-N.; Ren, D.-M.; Lou, H.-X.; Shen, T. Investigation of constituents from Cinnamomum camphora (L.) J. Presl and evaluation of their anti-inflammatory properties in lipopolysaccharide-stimulated RAW 264.7 macrophages. J. Ethnopharmacol. 2018, 221, 37-47. [CrossRef] 
14. Chen, Y.; Dai, G. Antifungal activity of plant extracts against Colletotrichum lagenarium, the causal agent of anthracnose in cucumber. J. Sci. Food Agric. 2012, 92, 1937-1943. [CrossRef]

15. Cha, J.D.; Jeong, M.R.; Jeong, S.I.; Moon, S.E.; Kil, B.S.; Yun, S.I.; Lee, K.Y.; Song, Y.H. Chemical composition and antimicrobial activity of the essential oil of Cryptomeria japonica. Phytother. Res. 2007, 21, 295-299. [CrossRef]

16. Shyur, L.-F.; Huang, C.-C.; Lo, C.-P.; Chiu, C.-Y.; Chen, Y.-P.; Wang, S.-Y.; Chang, S.-T. Hepatoprotective phytocompounds from Cryptomeria japonica are potent modulators of inflammatory mediators. Phytochemistry 2008, 69, 1348-1358. [CrossRef] [PubMed]

17. Cheng, S.-S.; Lin, H.-Y.; Chang, S.-T. Chemical composition and antifungal activity of essential oils from different tissues of Japanese cedar (Cryptomeria japonica). J. Agric. Food Chem. 2005, 53, 614-619. [CrossRef]

18. Kim, S.; Lee, S.; Hong, C.; Gwak, K.; Park, M.; Smith, D.; Choi, I. Whitening and antioxidant activities of bornyl acetate and nezukol fractionated from Cryptomeria japonica essential oil. Int. J. Cosmet. Sci. 2013, 35, 484-490. [CrossRef] [PubMed]

19. Tanase, C.; Domokos, E.; Cosarca, S.; Miklos, A.; Imre, S.; Domokos, J.; Dehelean, C.A. Study of the ultrasound-assisted extraction of polyphenols from beech (Fagus sylvatica L.) bark. Bioresources 2018, 13, 2247-2267. [CrossRef]

20. Jeong, K.M.; Ko, J.; Zhao, J.; Jin, Y.; Yoo, D.E.; Han, S.Y.; Lee, J. Multi-functioning deep eutectic solvents as extraction and storage media for bioactive natural products that are readily applicable to cosmetic products. J. Clean Prod. 2017, 151, 87-95. [CrossRef]

21. Dai, Y.; Verpoorte, R.; Choi, Y.H. Natural deep eutectic solvents providing enhanced stability of natural colorants from safflower (Carthamus tinctorius). Food Chem. 2014, 159, 116-121. [CrossRef] [PubMed]

22. The Commission of the European Communities. Commission Decision of 9 February 2006 amending Decision 96/335/EC establishing an inventory and a common nomenclature of ingredients employed in cosmetic products. Off. J. Eur. Union 2006, 257, 1-528.

23. Yoo, D.E.; Jeong, K.M.; Han, S.Y.; Kim, E.M.; Jin, Y.; Lee, J. Deep eutectic solvent-based valorization of spent coffee grounds. Food Chem. 2018, 255, 357-364. [CrossRef]

24. Dai, Y.; van Spronsen, J.; Witkamp, G.-J.; Verpoorte, R.; Choi, Y.H. Natural deep eutectic solvents as new potential media for green technology. Anal. Chim. Acta 2013, 766, 61-68. [CrossRef] [PubMed]

25. Li, R.; Yuan, C.; Dong, C.; Shuang, S.; Choi, M.M. In vivo antioxidative effect of isoquercitrin on cadmium-induced oxidative damage to mouse liver and kidney. Naunyn Schmiedebergs Arch. Pharmacol. 2011, 383, 437-445. [CrossRef]

26. Kim, Y.; Narayanan, S.; Chang, K.-O. Inhibition of influenza virus replication by plant-derived isoquercetin. Antivir. Res. 2010, 88, 227-235. [CrossRef]

27. Ma, C.; Jiang, Y.; Zhang, X.; Chen, X.; Liu, Z.; Tian, X. Isoquercetin ameliorates myocardial infarction through anti-inflammation and anti-apoptosis factor and regulating TLR4-NF-кB signal pathway. Mol. Med. Rep. 2018, 17, 6675-6680. [CrossRef]

28. Chatatikun, M.; Chiabchalard, A. Thai plants with high antioxidant levels, free radical scavenging activity, anti-tyrosinase and anti-collagenase activity. BMC Complement. Altern. Med. 2017, 17, 487. [CrossRef] [PubMed]

29. Sundaram, I.K.; Sarangi, D.D.; Sundararajan, V.; George, S.; Mohideen, S.S. Poly herbal formulation with anti-elastase and anti-oxidant properties for skin anti-aging. BMC Complement. Altern. Med. 2018, 18, 33.

30. Handa, C.L.; de Lima, F.S.; Guelfi, M.F.G.; Georgetti, S.R.; Ida, E.I. Multi-response optimisation of the extraction solvent system for phenolics and antioxidant activities from fermented soy flour using a simplex-centroid design. Food Chem. 2016, 197, 175-184. [CrossRef]

31. Bau, T.R.; Garcia, S.; Ida, E.I. Optimization of a fermented soy product formulation with a kefir culture and fiber using a simplex-centroid mixture design. Int. J. Food Sci. Nutr. 2013, 64, 929-935. [CrossRef] [PubMed]

(C) 2019 by the authors. Licensee MDPI, Basel, Switzerland. This article is an open access article distributed under the terms and conditions of the Creative Commons Attribution (CC BY) license (http://creativecommons.org/licenses/by/4.0/). 